\title{
Performance Evaluation of Generalized Polynomial Chaos
}

\author{
Dongbin Xiu, Didier Lucor, C.-H. Su, and George Em Karniadakis ${ }^{1}$ \\ Division of Applied Mathematics, Brown University, Providence, RI 02912, USA, \\ gk@dam. brown.edu
}

\begin{abstract}
In this paper we review some applications of generalized polynomial chaos expansion for uncertainty quantification. The mathematical framework is presented and the convergence of the method is demonstrated for model problems. In particular, we solve the first-order and second-order ordinary differential equations with random parameters, and examine the efficiency of generalized polynomial chaos compared to Monte Carlo simulations. It is shown that the generalized polynomial chaos can be orders of magnitude more efficient than Monte Carlo simulations when the dimensionality of random input is low, e.g. for correlated noise.
\end{abstract}

\section{Introduction}

The generalized polynomial chaos, also called the Wiener-Askey polynomial chaos, was first proposed in [6] for solving stochastic differential equations. It is a generalization of the classical Wiener's polynomial chaos, which is defined as the span of Hermite polynomial functionals of a Gaussian process [4]. The Hermite-chaos expansion converges to any $L^{2}$ functional in the $L^{2}$ sense, according to the Cameron-Martin theorem [1]. It has been a useful tool in the study of multiple Itô integrals. In recent years, the Wiener's Hermite-chaos has been applied to the stochastic modeling of engineering applications, including various problems in mechanics [2,3]. The more general framework of polynomial chaos employs many classes of orthogonal polynomials, and includes the Hermite-chaos as a subset. The main advantage of the generalized polynomial chaos is that it can represent many non-Gaussian stochastic processes, including some discrete processes, more efficiently [5-8].

In this paper, we review the theory and application of generalized polynomial chaos, and evaluate its performance by solving stochastic ordinary differential equations. 


\section{The Generalized Polynomial Chaos}

The generalized polynomial chaos is a means of representing second-order stochastic processes $X(\omega)$, viewed as a function of $\omega$ (the random event)

$$
\begin{aligned}
X(\omega) & =a_{0} \Psi_{0} \\
& +\sum_{i_{1}=1}^{\infty} a_{i_{1}} \Psi_{1}\left(\xi_{i_{1}}(\omega)\right) \\
& +\sum_{i_{1}=1}^{\infty} \sum_{i_{2}=1}^{i_{1}} a_{i_{1} i_{2}} \Psi_{2}\left(\xi_{i_{1}}(\omega), \xi_{i_{2}}(\omega)\right) \\
& +\sum_{i_{1}=1}^{\infty} \sum_{i_{2}=1}^{i_{1}} \sum_{i_{3}=1}^{i_{2}} a_{i_{1} i_{2} i_{3}} \Psi_{3}\left(\xi_{i_{1}}(\omega), \xi_{i_{2}}(\omega), \xi_{i_{3}}(\omega)\right) \\
& +\cdots
\end{aligned}
$$

where $\Psi_{n}\left(\xi_{i_{1}}, \ldots, \xi_{i_{n}}\right)$ denotes the generalized polynomial chaos of order $n$ in the variables $\left(\xi_{i_{1}}, \ldots, \xi_{i_{n}}\right)$, and are orthogonal polynomials in terms of the multidimensional random variables $\boldsymbol{\xi}=\left(\xi_{i_{1}}, \ldots, \xi_{i_{n}}\right)$. For notational convenience, one can re-arrange the terms in equation (1), according to some numbering scheme, and rewrite the expansion as

$$
X(\omega)=\sum_{j=0}^{\infty} \hat{a}_{j} \Phi_{j}(\boldsymbol{\xi})
$$

where there is a one-to-one correspondence between the functions $\Psi_{n}\left(\xi_{i_{1}}, \ldots, \xi_{i_{n}}\right)$ and $\Phi_{j}(\boldsymbol{\xi})$, and their corresponding coefficients $a_{i_{1} i_{2} i_{3} \ldots}$ and $\hat{a}_{j}$. Again $\left\{\Phi_{j}(\boldsymbol{\xi})\right\}$ are the (multi-dimensional) orthogonal polynomials in terms of the multi-dimensional random vector $\boldsymbol{\xi}$, satisfying the orthogonality relation

$$
\left\langle\Phi_{i}, \Phi_{j}\right\rangle=\left\langle\Phi_{i}^{2}\right\rangle \delta_{i j}
$$

where $\delta_{i j}$ is the Kronecker delta and $\langle\cdot, \cdot\rangle$ denotes the ensemble average. This is the inner product in the Hilbert space determined by the support of the random variables

$$
\langle f(\boldsymbol{\xi}), g(\boldsymbol{\xi})\rangle=\int f(\boldsymbol{\xi}) g(\boldsymbol{\xi}) w(\boldsymbol{\xi}) d \boldsymbol{\xi}
$$

with $w(\boldsymbol{\xi})$ denoting the weighting function. In the discrete case, the above orthogonal relation takes the form

$$
\langle f(\boldsymbol{\xi}), g(\boldsymbol{\xi})\rangle=\sum_{\boldsymbol{\xi}} f(\boldsymbol{\xi}) g(\boldsymbol{\xi}) w(\boldsymbol{\xi}) .
$$

In (2), there is a one-to-one correspondence between the type of the orthogonal polynomials $\{\Phi\}$ and the type of the random variables $\boldsymbol{\xi}$. This is determined by choosing the type of orthogonal polynomials $\{\Phi\}$ in such a way that 
their weighting function $w(\boldsymbol{\xi})$ in the orthogonality relation (4) has the same form as the probability distribution function of the underlying random variables $\boldsymbol{\xi}$. For example, the weighting function of Hermite orthogonal polynomials is $\frac{1}{\sqrt{(2 \pi)^{n}}} \exp \left(-\frac{1}{2} \boldsymbol{\xi}^{T} \boldsymbol{\xi}\right)$, and is the same as the probability density function of the $n$-dimensional Gaussian random variables $\boldsymbol{\xi}$. Hence, the classical Wiener polynomial chaos is an expansion of Hermite polynomials in terms of Gaussian random variables. Some types of generalized polynomial chaos corresponding to the commonly known distributions are listed in table 1.

Table 1. Correspondence of the type of Wiener-Askey polynomial chaos and their underlying random variables ( $N \geq 0$ is a finite integer).

\begin{tabular}{|c|c|c|c|}
\hline & Random variables $\boldsymbol{\xi}$ & Wiener-Askey chaos $\{\Phi(\boldsymbol{\xi})\}$ & Support \\
\hline \hline Continuous & Gaussian & Hermite-chaos & $(-\infty, \infty)$ \\
& gamma & Laguerre-chaos & {$[0, \infty)$} \\
& beta & Jacobi-chaos & {$[a, b]$} \\
& uniform & Legendre-chaos & {$[a, b]$} \\
\hline Discrete & Poisson & Charlier-chaos & $\{0,1,2, \ldots\}$ \\
& binomial & Krawtchouk-chaos & $\{0,1, \ldots, N\}$ \\
& negative binomial & Meixner-chaos & $\{0,1,2, \ldots\}$ \\
& hypergeometric & Hahn-chaos & $\{0,1, \ldots, N\}$ \\
\hline
\end{tabular}

The expansion (1) (or (2)) resides in the infinite dimensional space determined by $\boldsymbol{\xi}$, and is an infinite summation. In practice, we have to restrict ourselves to the finite-term summation. This is achieved by reducing the expansion to the finite-dimensional space, i.e. expansion of finite-dimensional random variables $\boldsymbol{\xi}$, according to the nature of random inputs; we also set the highest order of the polynomials $\{\Phi\}$ according to accuracy requirement. The finite-term expansion takes the form

$$
X(\omega)=\sum_{j=0}^{M} \hat{a}_{j} \Phi_{j}(\boldsymbol{\xi}),
$$

where $\boldsymbol{\xi}$ is an $n$-dimensional random vector. If the highest order of polynomial $\{\Phi\}$ is $m$, then the total number of expansion terms $(M+1)$ is, $(M+1)=$ $(n+m) ! /(n ! m !)$.

\section{Applications to Stochastic ODEs}

In this section we apply the generalized polynomial chaos to the solution of stochastic ordinary differential equations with random parameters. We first consider the first-order ODE and demonstrate in detail the solution procedure; we then consider a second-order ODE with multiple random parameters which results in multi-dimensional polynomial chaos expansion. Hereafter, we restrict our discussion to the continuous probability distributions. 


\subsection{First-Order ODE}

We consider the ordinary differential equation

$$
\frac{d y(t)}{d t}=-k y, \quad y(0)=\hat{y}
$$

where the decay rate coefficient $k$ is considered to be a random variable $k(\omega)$ with certain probability density function (PDF) $f(k)$, zero mean value and standard deviation $\sigma_{k}$.

By applying the Wiener-Askey polynomial chaos expansion (6) to the solution $y$ and random input $k$

$$
y(t)=\sum_{i=0}^{M} y_{i}(t) \Phi_{i}, \quad k=\sum_{i=0}^{M} k_{i} \Phi_{i}
$$

and substituting the expansions into the governing equation, we obtain

$$
\sum_{i=0}^{M} \frac{d y_{i}(t)}{d t} \Phi_{i}=-\sum_{i=0}^{M} \sum_{j=0}^{M} \Phi_{i} \Phi_{j} k_{i} y_{j}(t) .
$$

We then project the above equation onto the random space spanned by the orthogonal polynomial basis $\left\{\Phi_{i}\right\}$ by taking the inner product of the equation with each basis. By taking $<., \Phi_{l}>$ and utilizing the orthogonality condition (3), we obtain the following set of equations:

$$
\frac{d y_{l}(t)}{d t}=-\frac{1}{\left\langle\Phi_{l}^{2}\right\rangle} \sum_{i=0}^{M} \sum_{j=0}^{M} e_{i j l} k_{i} y_{j}(t), \quad l=0,1, \ldots, M
$$

where $e_{i j l}=\left\langle\Phi_{i} \Phi_{j} \Phi_{l}\right\rangle$. Note that the coefficients are smooth and thus any standard ODE solver can be employed here, e.g. Rouge-Kutta methods.

In Figure 1 we show the error convergence at time $t=1$ of the Hermitechaos expansion and Jacobi-chaos expansion, subject to Gaussian input and beta input, respectively. It can be seen on the semi-log plot that the errors decay exponentially fast as the highest order of expansion $(P)$ increases. Given the simplicity of the equation, we can estimate the solution error of the finiteterm chaos expansion. In particular, we estimate analytically the relative error in variance, denoted $\epsilon_{2}(t)$, for different distributions of $k$ as a function of time $[9]$ :

$$
\begin{aligned}
& \epsilon_{2}(t)=\left(\frac{\sigma t}{1+\sigma t}\right)^{2(M-1)}, \\
& \epsilon_{2}(t) \leq \frac{(\sigma t)^{2(M+1)}}{e^{(\sigma t)^{2}}-1}\left[(M+1) !\left(1-\frac{(\sigma t)^{2}}{M+1}\right)\right],
\end{aligned}
$$

for the Laguerre-chaos when $k$ has exponetial distribution and the Hermitechaos when $k$ has Gaussian distribution, respectively. Similar estimates can 

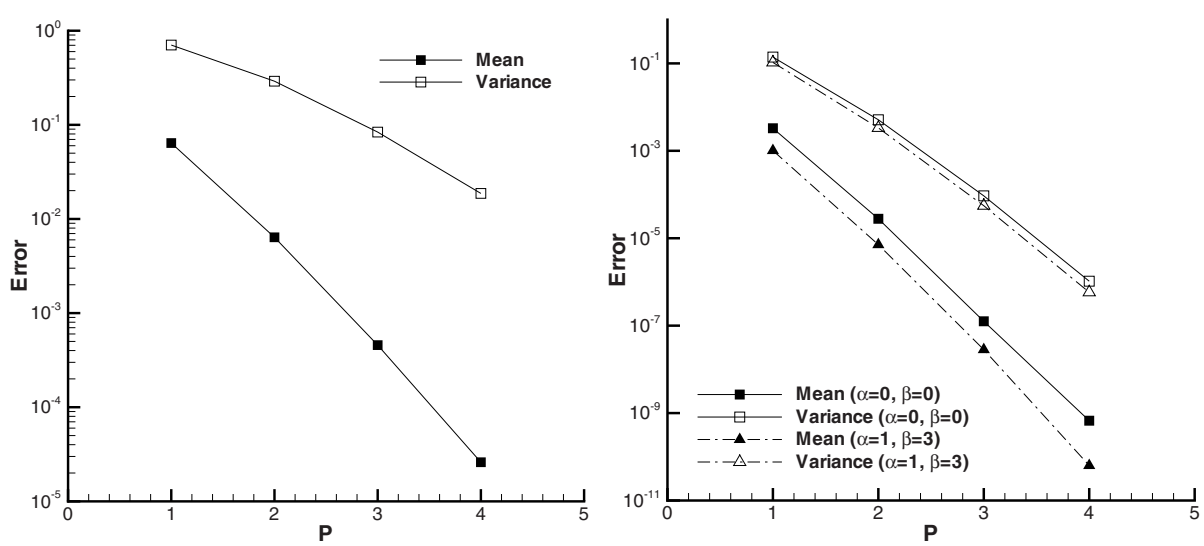

Fig. 1. Error convergence of the mean and the variance $\left(\sigma_{k}=1\right)$. Left: Hermite-chaos with Gaussian random input, Right: Jacobi-chaos with beta random input.

be applied to Legendre-chaos with uniform random input, although no explicit analytical formula is available and the estimation has to be evaluated numerically. By using these error estimates, we examine the number of expansion terms needed for a given error control threshold. The results of Hermite-chaos with Gaussian input and Legendre-chaos with uniform input are plotted in Figure 2, for fixed relative error of $10^{-7}$ in variance. It can be seen that the number of Hermite-chaos needed is larger than Legendre-chaos, and grows faster over time. For details of these estimates, see [9].

\subsection{Second-Order ODE}

In this section, we consider a linear oscillator subject to both random parametric and external forcing excitations, in the form

$$
\begin{aligned}
& \ddot{x}(t)+2 \zeta \omega_{0} \dot{x}(t)+\omega_{0}^{2} x(t)=F(\omega) \cos (\omega t+\phi), \\
& x(0)=x_{0}, \quad \dot{x}(0)=\dot{x}_{0}, \quad t \in[0, T]
\end{aligned}
$$

We assume the parameters and the forcing amplitude are random variables, i.e.

$$
\begin{aligned}
c & \doteq 2 \zeta \omega_{0}=\bar{c}+\sigma_{c} \xi_{1} \\
k & \doteq \omega_{0}^{2}=\bar{k}+\sigma_{k} \xi_{2} \\
F & =\bar{F}+\sigma_{F} \xi_{3},
\end{aligned}
$$



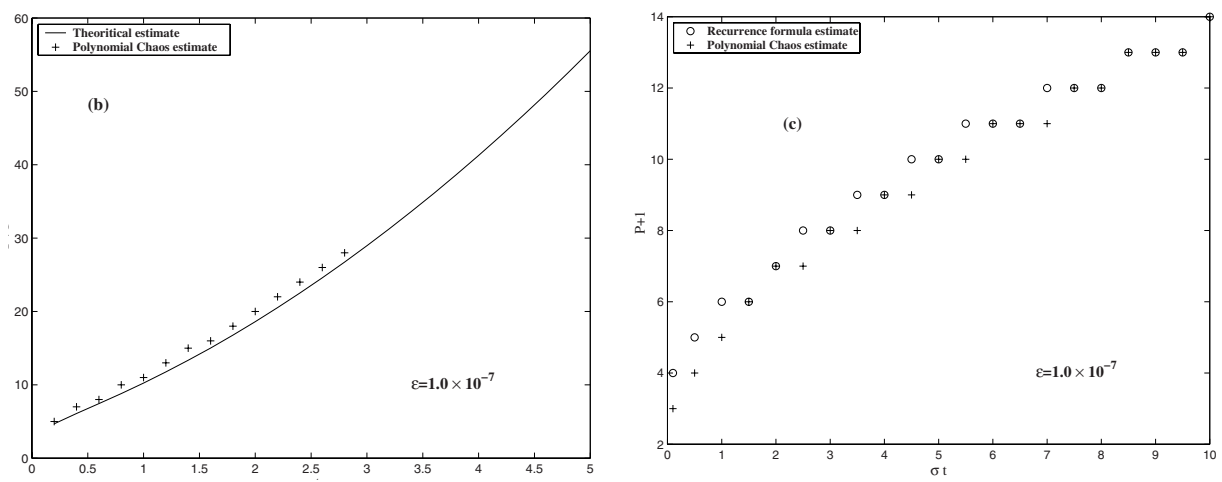

Fig. 2. Number of expansion terms needed for given relative error in variance $\epsilon_{2}=$ $10^{-7}$. Left: Hermite-chaos with Gaussian random input, Right: Legendre-chaos with uniform random input.

where $\xi_{1}, \xi_{2}$ and $\xi_{3}$ are three independent random variables with zero mean; $\sigma_{c}$, $\sigma_{k}$ and $\sigma_{F}$ scales as the standard deviations of $c, k$ and $F$, respectively. Here we will consider the uniform and Gaussian distributions. Correspondingly, the Legendre-chaos and Hermite-chaos will be employed.

The numerical integration is performed up to $T=100$ when the solution reaches the asymptotic periodic state. We examine the convergence of the relative error in mean and variance at the final time versus the expansion order(see figure 3 ). It can be seen that the errors of the mean and variance decrease exponentially fast as the expansion order increases. However, in the Legendre-chaos the error in the mean and variance decay at the same rate in contrast to the Hermite-chaos.

\section{Efficiency}

The expanded equations from generalized polynomial chaos is a set of coupled equations, with each one of them resembles the deterministic equation. Since these equations are solved explicitly, the overall computational cost of generalized polynomial expansion is roughly the number of expansion terms times the cost of a single deterministic solution. On the other hand, the cost of Monte Carlo simulations is the number of realizations times that of a deterministic solution. Normally, thousands of realizations are needed for Monte Carlo simulation to obtain the solution statistics with moderate accuracy. Thus, the generalized 

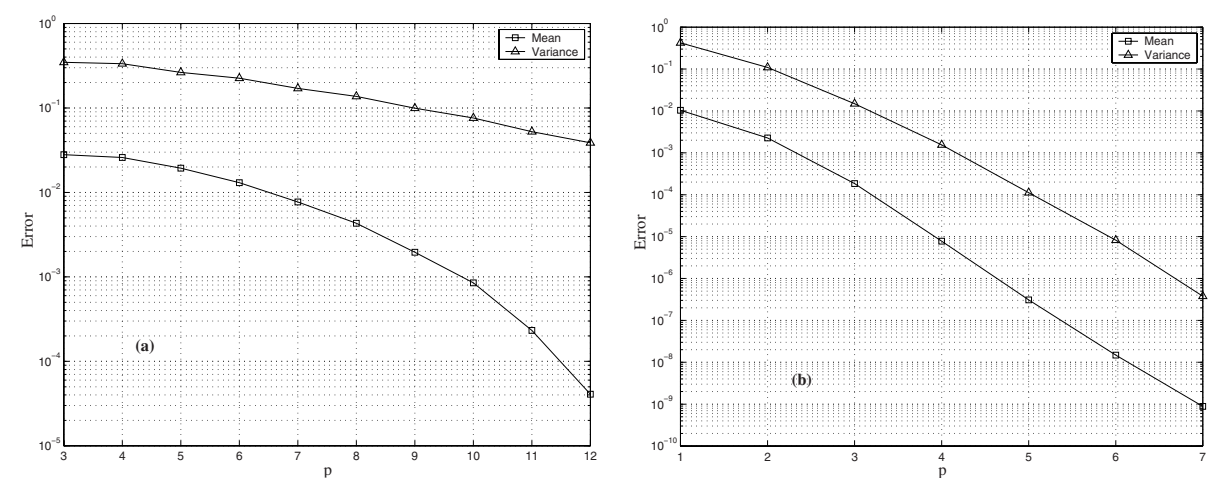

Fig. 3. Error convergence of the mean and variance. Left: Hermite-chaos with Gaussian random input; Right: Legendre-chaos with uniform random input.

polynomial chaos offers a great potential of computational speed-up due to its fast convergence rate.

For example, if $k$ is an exponentially distributed random variable in the firstorder ODE problem discussed in 3.1, the error convergence of the mean solution of the Monte-Carlo simulation and the corresponding Laguerre-chaos is shown in table 2 .

Table 2. Speed-up factors $S$ based on the relative error in mean $\left(\epsilon_{\text {mean }}\right)$ with exponential random input, for the first-order ODE problem $(7)$. ( $S$ is defined as the cost of Monte Carlo simulations over that of generalized polynomial chaos.)

\begin{tabular}{|c|c|c|c|c|}
\hline & $\epsilon_{\text {mean }}$ & $\begin{array}{c}\text { Monte Carlo simulation } \\
\text { (no. of realizations) }\end{array}$ & $\begin{array}{c}\text { Generalized polynomial chaos: } \\
\text { (no. of expansion terms) }\end{array}$ & $S$ \\
\hline \multirow{3}{*}{ Exponential } & $4 \%$ & 100 & 1 & 100 \\
& $1.1 \%$ & 1,000 & 2 & 500 \\
& $0.05 \%$ & 9,800 & 3 & 3,267 \\
\hline
\end{tabular}

For the second-order linear oscillator (13), we examine the efficiency of generalized polynomial chaos and Monte Carlo simulation by fixing the error requirement in mean at $T=100$. Both uniform random inputs and Gaussian random inputs are considered, and the results are summarized in table 3. Smaller speedup factors are observed for the Hermite-chaos expansion with Gaussian inputs, 
compared with Legendre-chaos with uniform inputs. The advantage of the exponential convergence of chaos expansion is obvious, especially when smaller errors are required. In the case of uniform random input with error requirement of $0.001 \%$ in mean, a speed-up factor of 17 millions is observed for Legendrechaos expansion.

Table 3. Speed-up factors $S$ based on the relative error in mean $\left(\epsilon_{\text {mean }}\right)$ with Gaussian and uniform random inputs, for the second-order ODE problem (13).

\begin{tabular}{|c|c|c|c|c|}
\hline & $\epsilon_{\text {mean }}$ & $\begin{array}{c}\text { Monte Carlo simulation } \\
\text { (no. of realizations) }\end{array}$ & $\begin{array}{c}\text { Generalized polynomial chaos: } \\
\text { (no. of expansion terms) }\end{array}$ & $S$ \\
\hline \multirow{3}{*}{ Gaussian } & $2 \%$ & 350 & 56 & 6.25 \\
& $0.8 \%$ & 2,150 & 120 & 18 \\
& $0.2 \%$ & 33,200 & 220 & 151 \\
\hline \multirow{3}{*}{ Uniform } & $0.2 \%$ & 13,000 & 10 & 13,000 \\
& $0.018 \%$ & $1,58 \times 10^{6}$ & 20 & 79,000 \\
& $0.001 \%$ & $6.1 \times 10^{8}$ & 35 & $1.7 \times 10^{7}$ \\
\hline
\end{tabular}

\section{Conclusion}

The efficiency of the generalized polynomial chaos can be orders of magnitude higher than the methods that require sampling, e.g. Monte Carlo method. This is especially obvious when the random inputs have low to moderate dimensions. However, if the random input has large dimensions, i.e. in the limit of white noise, the cost of generalized polynomial chaos grows fast, for a large number of expansion terms is required. In this case, the efficiency of the generalized polynomial chaos expansion is reduced drastically and the Monte Carlo method may be more efficient. This is still an unresolved problem for the generalized polynomial chaos and more work is required to resolve this issue.

\section{Acknowledgements.}

This work was supported by the ITR-DDDAS program of the National Science Foundation.

\section{References}

1. Cameron, R., Martin, W.: The orthogonal development of nonlinear functionals in series of Fourier-Hermite functionals. Ann. Math. 48 (1947) 385-392

2. Ghanem, R.: Ingredients for a general purpose stochastic finite element formulation. Comput. Methods Appl. Mech. Engrg. 168 (1999) 19-34 
3. Ghanem, R., Spanos, P.: Stochastic Finite Elements: a Spectral Approach. SpringerVerlag, 1991

4. Wiener, N.: The homogeneous chaos. Amer. J. Math., 60 (1938) 897-936

5. Xiu, D., Karniadakis, G.: Modeling uncertainty in steady state diffusion problems via generalized polynomial chaos. Comput. Methods Appl. Math. Engrg. 191 (2002) 4927-4948

6. Xiu, D., Karniadakis, G.: The Wiener-Askey polynomial chaos for stochastic differential equations. SIAM J. Sci. Comput. 24 (2002) 619-644

7. Xiu, D.,Karniadakis, G.: Modeling uncertainty in flow simulations via generalized polynomial chaos. J. Comput. Phys. (to appear)

8. Xiu, D., Lucor, D., Su, C., Karniadakis, G.: Stochastic modeling of flow-structure interactions using generalized polynomial chaos. J. Fluids Eng. 124 (2002) 51-59

9. Lucor, D., Su., C., Karniadakis, G.: Generalized polynomial chaos and random oscillators. Int. J. Numer. Methods Eng. (submitted) 